\title{
Integrability and disorder in mesoscopic systems: Application to orbital magnetism
}

\author{
Klaus Richter \\ Institut für Physik, Memminger Strasse. 6, 86135 Augsburg, Germany \\ and Max-Planck-Institut für Physik komplexer Systeme, 01187 Dresden, Germany \\ Denis Ullmo a) \\ Bell Laboratories, Lucent Technologies, 1D-265, \\ 600 Mountain Avenue, Murray Hill, New Jersey 07974-0636
}

Rodolfo A. Jalabert

Université Louis Pasteur, IPCMS-GEMME, 23 rue du Loess,

67037 Strasbourg Cedex, France

(Received 23 April 1996; accepted for publication 19 June 1996)

We present a semiclassical theory of weak disorder effects in small structures and apply it to the magnetic response of non-interacting electrons confined in integrable geometries. We discuss the various averaging procedures describing different experimental situations in terms of one- and two-particle Green functions. We demonstrate that the anomalously large zero-field susceptibility characteristic of clean integrable structures is only weakly suppressed by disorder. This damping depends on the ratio of the typical size of the structure with the two characteristic length scales describing the disorder (elastic mean-free-path and correlation length of the potential) in a power-law form for the experimentally relevant parameter region. We establish the comparison with the available experimental data and we extend the study of the interplay between disorder and integrability to finite magnetic fields. (C) 1996 American Institute of Physics. [S0022-2488(96)01310-2]

\section{INTRODUCTION}

Electronic mesoscopic systems offer nowadays the possibility of being used as a laboratory for studying quantum chaos. The main question of this novel discipline - the quantum signatures of the underlying classical dynamics - can be addressed in microstructures defined on high mobility semiconductor heterojunctions. This connection presents a considerable challenge to experimentalists since it implies complicated fabrication processes and delicate measurements. The challenge for theoreticians is not any less complicated since semiconductor microstructures are very rich condensed matter systems (involving effects of temperature, confinement, disorder, electron-electron and electron-phonon interactions, etc.) where the applicability and validity of simple models has to be clearly established.

Within the simple model of a particle-in-a-billiard, important differences have been predicted, ${ }^{1}$ and later measured, ${ }^{2,3}$ in the transport through chaotic and integrable geometries. In the former nearby trajectories diverge exponentially and periodic orbits are usually isolated; the latter are characterized by having as many constants of motion in involution as degrees of freedom, and periodic orbits are organized in families on invariant tori. ${ }^{4}$ Chaotic cavities exhibit a universal behavior for the conductance fluctuations and weak-localization, characterized by a single scale. On the contrary, integrable cavities do not show generic behavior presenting more fine-scale fluctuations and a non-Lorentzian line-shape of the low-field magneto resistance. In the case of thermodynamical properties like the magnetic susceptibility, the differences between chaotic and integrable billiards are more spectacular since they involve an order-of-magnitude enhancement of

aPermanent address: Division de Physique Théorique, IPN, 91406 Orsay Cedex, France. 
the low-field susceptibility of integrable geometries compared to that of chaotic ones. ${ }^{5-7}$ Unlike the transport problem, the predicted different behavior according to the integrability of the underlying classical mechanics, has not been experimentally confirmed.

The residual disorder present in actual microstructures plays a special role in the quantum chaos studies. Indeed, any perturbing potential, such as the one provided by the disorder, immediately breaks the integrable character of the classical dynamics. Since small amounts of disorder are unavoidable in actual microstructures, the question of whether or not integrable behavior should be observed, naturally arises. It is then of foremost importance to establish if the differences between chaotic and integrable geometries persist when we go beyond the particle-in-a-box model. This interplay between integrability and disorder is the main subject of this paper.

We start by characterizing the disorder. One limiting case is the absence of it, where the dynamics is determined by the non-random confinement potential (particle-in-a-box or clean models). On the other extreme we have the diffusive limit where the electron motion is a random walk between the impurities and the confining effects are not important. The strength of the disorder in the diffusive case is characterized by the transport mean free path $l_{T}$ : the mean distance over which the electron momentum is randomized. When $l_{T}$ becomes of the order of the typical size $a$ of the microstructure, confinement and disorder are relevant. For $l_{T}>a$ we arrive at the ballistic regime where electrons can traverse the structure with a small drift in their momentum (going along almost straight lines), and their dynamics is mainly given by the bounces off the walls of the confining potential. In the ballistic regime the underlying classical mechanics still depends on the geometry and we would like to understand the different role of disorder in integrable and chaotic geometries.

For short range impurity potentials (as typically found in metallic samples) the scattering is isotropic ( $s$-type) and the momentum is randomized after each collision with an impurity. There is therefore only one length scale, namely $l_{T}$, characterizing the disorder. For smooth impurity potentials (as typically realized in high-mobility microstructures) the scattering is forward directed and $l_{T}$ may be significantly larger than the elastic mean free path $l$ associated to the total amplitude diffracted by the disorder. ${ }^{8}$ The regime $l_{T}>a>l$ is particularly interesting because it is ballistic (since the classical mechanics is hardly affected by disorder), but the single particle eigenstates are short lived. In a more technical language that we will precise in the sequel, we have $l$ given by a single-particle Green function and $l_{T}$ by a two-particle Green function. ${ }^{9}$ We will study the interplay between disorder and confinement for physical observables that depend on one- and two-particle Green functions, concentrating on the magnetic susceptibility of individual and ensembles of ballistic microstructures.

The natural tools to attack the interplay between disorder and confinement are semiclassical expansions since they transparently convey at the quantum level the information about the classical mechanics. Supersymmetry ${ }^{10}$ and random matrix theories are quite powerful methods that have been widely used in recent studies of quantum chaos and disordered systems, ${ }^{11-14}$ but are not applicable to our regime of interest since they deal with the ergodic universal (long time) properties of completely chaotic systems. Diagrammatic perturbation theory for the disorder can describe the diffusive regime, ${ }^{15}$ but calculations become exceedingly complicated when the confinement and the detailed nature of the impurity potential has to be considered.

In our semiclassical approach we emphasize the dependence of disorder effects on the ratio between the finite system size $a$ and the disorder correlation length $\xi$, showing that confined systems exhibit strong deviations from the bulk-behavior. In particular we demonstrate that for integrable geometries the effect of smooth disorder results in a power-law damping of the twoparticle Green function properties, and we compare this behavior with that expected in chaotic systems. For completeness of the presentation we first briefly review in Sec. II our work on the magnetic response of clean systems. ${ }^{5,6}$ We then develop in detail a treatment of disorder in ballistic microstructures extending some preliminary work. ${ }^{16}$ In Sec. III we present the disorder model and some general implications at the level of one- and two-particle Green functions. In 
Secs. IV and V we focus on the impurity averaged magnetic susceptibility for individual and ensembles of microstructures.

\section{ORBITAL MAGNETISM IN CLEAN SYSTEMS: A BRIEF REVIEW}

\section{A. Thermodynamic formalism}

In this section we present the basic thermodynamical formalism for obtaining the orbital magnetism within a semiclassical approach. We indicate the main ideas for its application to clean microstructures $^{5,6}$ which will be further developed in Secs. IV and V in order to allow for the treatment of static disorder. The principle is to derive thermodynamical expressions for the free energy and the grand potential using a semiclassical approximation for the density of states. This allows us to calculate physical observables such as the magnetic susceptibility for the canonical and grand canonical ensembles.

For a system of electrons confined to an area $A$ at temperature $T$ and subject to a perpendicular magnetic field $H$, the free energy $F(T, H, \mathbf{N})$ for a fixed number $\mathbf{N}$ of electrons and the grand potential $\Omega(T, H, \mu)$ (representing the coupling to a particle reservoir with chemical potential $\mu)$ are related by means of the Legendre transform

$$
F(T, H, \mathbf{N})=\mu \mathbf{N}+\Omega(T, H, \mu) .
$$

The canonical $(\chi)$ and grand canonical $\left(\chi^{\mathrm{GC}}\right)$ susceptibilities of a confined electron gas are given by

$$
\chi=-\frac{1}{A}\left(\frac{\partial^{2} F}{\partial H^{2}}\right)_{T, \mathbf{N}}, \quad \chi^{\mathrm{GC}}=-\frac{1}{A}\left(\frac{\partial^{2} \Omega}{\partial H^{2}}\right)_{T, \mu} .
$$

The grand potential can be expressed in the form

$$
\Omega(T, H, \mu)=-\frac{1}{\beta} \int \mathrm{d} E d(E) \ln [1+\exp (\beta(\mu-E))]
$$

(with $\beta=1 / k_{B} T$ ) in terms of the single-particle density of states $d(E)$ which we decompose into a smooth mean and oscillating part according to

$$
d(E)=\bar{d}(E)+d^{\mathrm{osc}}(E) .
$$

As has first been noticed in the context of persistent currents in disordered rings, ${ }^{17}$ a distinction between $\chi$ and $\chi^{\mathrm{GC}}$ may be of crucial importance in mesoscopic thermodynamics: Although the number of electrons can be large for a mesoscopic system, the fact that $\mathbf{N}$ is fixed must be taken into account (by working in the canonical formalism) if a disorder or energy averaged magnetic response of an ensemble of isolated microsystems is examined. According to $\operatorname{Imry}^{18}$ a convenient representation for the canonical free energy in terms of grand canonical quantities is obtained by expanding the relationship (2.1) to second order in $\mu-\bar{\mu}$ with a mean chemical potential $\bar{\mu}$ being implicitly defined by accommodating $\mathbf{N}$ charge carriers with the mean number of states

$$
\mathbf{N}=N(\mu)=\bar{N}(\bar{\mu}) .
$$

Here

$$
N(\mu)=\int_{0}^{\infty} \mathrm{d} E d(E) f(E-\mu)
$$

with the Fermi distribution function 


$$
f(E-\mu)=\frac{1}{1+\exp [\beta(E-\mu)]}
$$

$\bar{N}$ is obtained in Eq. (2.5) by replacing $d(E)$ by $\bar{d}(E)$. This finally allows an expansion of the free energy as ${ }^{17}$

$$
F(\mathbf{N}) \simeq F^{0}+\Delta F^{(1)}+\Delta F^{(2)}
$$

with

$$
\begin{gathered}
F^{0}=\bar{\mu} \mathbf{N}+\bar{\Omega}(\bar{\mu}), \\
\Delta F^{(1)}=\Omega^{\mathrm{osc}}(\bar{\mu}), \\
\Delta F^{(2)}=\frac{1}{2 \bar{d}(\bar{\mu})}\left(N^{\mathrm{osc}}(\bar{\mu})\right)^{2} .
\end{gathered}
$$

The functions $\Omega^{\text {osc }}(\bar{\mu})$ and $N^{\text {osc }}(\bar{\mu})$ are expressed by means of Eqs. (2.3) and (2.6), respectively, upon inserting the oscillating part $d^{\text {osc }}(E)$ of the density of states (2.4). The leading order contribution to $F$ is given by the first two terms $F^{0}+\Delta F^{(1)}$ yielding the susceptibility calculated in the grand canonical case. $F^{0}$ gives rise to the (two-dimensional) diamagnetic Landau-susceptibility which for billiard-like systems is expressed as for the bulk as

$$
-\chi_{L}=-\frac{g_{s} e^{2}}{24 \pi m c^{2}}
$$

with $g_{s}=2$ the spin degeneracy.

\section{B. Semiclassical treatment of susceptibilities}

For a semiclassical computation of $\Delta F^{(1)}$ and $\Delta F^{(2)}$ and their derivatives with respect to $H$ we calculate $d^{\text {osc }}(E, H)$ from the trace

$$
d(E, H)=-\frac{\mathrm{g}_{\mathrm{s}}}{\pi} \operatorname{Im} \int d \mathbf{r} G_{E}(\mathbf{r}, \mathbf{r})
$$

of the semiclassical one-particle Green function. Its contribution to $d^{\text {osc }}(E)$ is given by ${ }^{4}$

$$
G_{E}\left(\mathbf{r}^{\prime}, \mathbf{r}\right)=\sum_{t} D_{t} \exp \left[i\left(\frac{S_{t}}{\hbar}-\eta_{t} \frac{\pi}{2}\right)\right],
$$

as the sum over all classical paths $t$ (of non-zero length) joining $\mathbf{r}$ to $\mathbf{r}^{\prime}$ at energy $E$.

$$
S_{t}=\int_{\mathscr{C}_{t}} \mathbf{p} d \mathbf{q}
$$

is the classical action integral along the path $\mathscr{C}_{t}$. The amplitude $D_{t}$ takes care of the classical probability conservation, and $\eta_{t}$ is the Maslov index.

The evaluation of the trace integral (2.11) for chaotic and integrable systems leads to the Gutzwiller $^{4}$ and Berry-Tabor ${ }^{19}$ periodic-orbit trace formulas, respectively. In order to calculate the magnetic susceptibility at small fields one has to carefully distinguish ${ }^{6}$ between the three 
possibilities of a chaotic billiard, the special case of an integrable billiard remaining integrable upon inclusion of the $H$-field, and the more general case where the field acts as a perturbation breaking the integrability of a regular structure.

Since our main interest in Secs. III, IV and V will be devoted to disorder effects on the susceptibility of billiards being integrable at zero $H$-field we will focus here on the last case. There neither Gutzwiller nor Berry-Tabor-trace formulas are directly applicable and, following Ozorio de Almeida, ${ }^{20}$ a uniform treatment of the perturbing $H$-field is necessary. In the integrable zerofield limit each closed trajectory belongs to a torus $I_{\mathbf{M}}$ and we can replace $\mathbf{r}$ in the trace integral (2.11) by angle coordinates $\Theta_{1}$ specifying the trajectory within the (one-parameter) family and by the position $\Theta_{2}$ on the trajectory. For small magnetic field the classical orbits can be treated as essentially unaffected while the field acts merely on the phases in the Green function in terms of the magnetic flux through the area $\mathscr{C}_{\mathbf{M}}\left(\Theta_{1}\right)$ enclosed by each orbit of family $\mathbf{M}$. Evaluating the trace integral (2.11) along $\Theta_{2}$ for the semiclassical Green function of an integrable system leads in this approximation to a factorization of the density of states

$$
d^{\mathrm{osc}}(E)=\sum_{\mathbf{M} \neq 0} \mathscr{C}_{\mathbf{M}}(H) d_{\mathbf{M}}^{0}(E)
$$

into the contribution from the integrable zero-field limit

$$
d_{\mathbf{M}}^{0}(E)=\widetilde{D}_{\mathbf{M}} \cos \left(k_{F} L_{\mathbf{M}}-\eta_{\mathbf{M}} \frac{\pi}{2}-\frac{\pi}{4}\right)
$$

( $L_{\mathbf{M}}$ is the length of the orbits of family $\mathbf{M}$ and $\widetilde{D}_{\mathbf{M}}$ the semiclassical weight ${ }^{19}$ ) and the function

$$
\mathscr{C}_{\mathbf{M}}(H)=\frac{1}{2 \pi} \int_{0}^{2 \pi} \mathrm{d} \Theta_{1} \cos \left[2 \pi \frac{H \cdot \mathscr{C}_{\mathbf{M}}\left(\Theta_{1}\right)}{\Phi_{0}}\right]
$$

containing the $H$-field dependence $\left(\Phi_{0}=h c / e\right)$. Calculating $\Delta F^{(1)}$ from Eq. $(2.9 \mathrm{~b})$ and taking the derivatives with respect to $H$ gives the grand canonical contribution to the susceptibility at small magnetic field

$$
\frac{\chi^{(1)}}{\chi_{L}}=-\frac{24 \pi}{\mathrm{g}_{\mathbf{s}}} m A\left(\frac{\Phi_{0}}{2 \pi A}\right)^{2} \sum_{\mathbf{M}} \frac{R_{T}\left(\tau_{\mathbf{M}}\right)}{\tau_{\mathbf{M}}^{2}} d_{\mathbf{M}}^{0}(\mu) \frac{d^{2} \mathscr{C}_{\mathbf{M}}}{d H^{2}} .
$$

Here, $\tau_{\mathbf{M}}$ is the period of a closed orbit of family $\mathbf{M}$ and

$$
R_{T}(\tau)=\frac{\tau / \tau_{c}}{\sinh \left(\tau / \tau_{c}\right)} ; \quad \tau_{c}=\frac{\hbar \beta}{\pi}
$$

is a temperature damping factor which arises from the convolution integral in Eq. (2.3) and gives an exponential suppression of long orbits. This is important from a physical as well as computational point of view, as conceptual difficulties associated with the questions of absolute convergence of semiclassical expansions at zero temperature do not arise.

Eq. (2.17) is the basic equation for the susceptibility of an individual microstructure. When considering ensembles of ballistic microstructures however, an average $(\cdot)$ over energy (i.e., $k_{F}$ ) or over the system size $a$ usually has to be performed and leads to variations in the phases (actions $S / \hbar=k_{F} L_{\mathbf{M}}$ ) of the density of states (2.15) which are much larger than $2 \pi$. Therefore, $\chi^{(1)}$ vanishes upon ensemble average. In order to characterize the orbital magnetism of ensembles we introduce the typical susceptibility $\chi^{(\mathrm{t})}=\left(\overline{\chi^{2}}\right)^{1 / 2}$ (the width of the distribution) and the ensemble average $\bar{\chi}$ [its mean value, which is non-zero because of the term $\Delta F^{(2)}$ in the expansion 


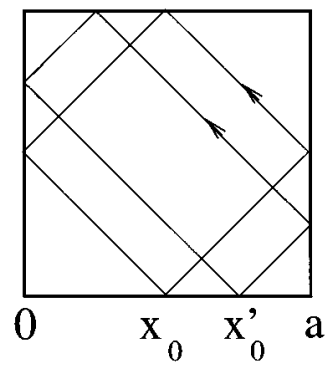

FIG. 1. Two representative periodic orbits characterized by $x_{0}$ and $x_{0}^{\prime}$ belonging to the family $\mathbf{M}=(1,1)$ (denoting one bounce with each wall) of a square billiard of length $a$.

Eq. (2.8)]. The typical and ensemble average susceptibilities are of theoretical interest since they are based on two-particle Green functions and are relevant for the description of experiments on ensembles of mesoscopic systems.

If we assume that there are no degeneracies in the lengths of orbits from different families M we obtain for $\chi^{(\mathrm{t})}$

$$
\left(\frac{\chi^{(\mathrm{t})}}{\chi_{L}}\right)^{2}=\left(\frac{24 \pi}{\mathrm{g}_{\mathrm{s}}} m A\right)^{2}\left(\frac{\Phi_{0}}{2 \pi A}\right)^{4} \sum_{\mathbf{M}} \frac{R_{T}^{2}\left(\tau_{\mathbf{M}}\right)}{\tau_{\mathbf{M}}^{4}} \overline{d_{\mathbf{M}}^{0}(\mu)^{2}}\left(\frac{d^{2} \mathscr{C}_{\mathbf{M}}}{d H^{2}}\right)^{2}
$$

In calculating $\bar{\chi}$, the grand canonical contribution $\chi^{(1)}$ from $\Delta F^{(1)}$ vanishes under energy average and the canonical correction $\Delta F^{(2)}$ in Eq. (2.8) gives in semiclassical approximation using Eq. $(2.9 \mathrm{c})$

$$
\frac{\bar{\chi}}{\chi_{L}} \simeq \frac{\overline{\chi^{(2)}}}{\chi_{L}}=-\frac{24 \pi^{2}}{g_{\mathbf{s}}^{2}} \hbar^{2}\left(\frac{\Phi_{0}}{2 \pi A}\right)^{2} \sum_{\mathbf{M}} \frac{R_{T}^{2}\left(\tau_{\mathbf{M}}\right)}{\tau_{\mathbf{M}}^{2}} \overline{d_{\mathbf{M}}^{0}(\mu)^{2}} \frac{d^{2} \mathscr{C}_{\mathbf{M}}^{2}}{d H^{2}} .
$$

Eqs. (2.17)-(2.20) provide the general starting point for a computation of the susceptibility of integrable billiards at small fields.

As an important example, which is also of experimental relevance, ${ }^{21}$ we will apply the results to square billiards. At finite temperature $\chi$ is essentially given by the family $\mathbf{M}=(1,1)$ of the shortest, flux-enclosing periodic orbits depicted in Fig. 1. A complete treatment including families of longer orbits is given in Ref. 6. Instead of $\Theta_{1}$ we use the lower reflection point $x_{0}$ as orbit parameterization within the family. The orbits $(1,1)$ have the unique length $L_{11}=2 \sqrt{2} a$ and enclose a normalized area $\mathscr{b}\left(x_{0}\right)=4 \pi x_{0}\left(a-x_{0}\right) / a^{2}$. Computation of $d_{11}^{0}(\mu)$ for the square geometry gives for $\chi^{(1)}$ [Eq. (2.17)]

$$
\frac{\chi^{(1)}}{\chi^{0}}=\int_{0}^{a} \frac{\mathrm{d} x_{0}}{a} \mathscr{C}^{2}\left(x_{0}\right) \cos \left(\varphi \mathscr{C}\left(x_{0}\right)\right) \sin \left(k_{F} L_{11}+\frac{\pi}{4}\right)
$$

as a function of the total flux $\varphi=H a^{2} / \Phi_{0}$ with $\Phi_{0}=h c / e$. The prefactor

$$
\chi^{0}=\chi_{L} \frac{3}{(\sqrt{2} \pi)^{5 / 2}}\left(k_{F} a\right)^{3 / 2} R_{T}\left(L_{11}\right)
$$

shows the $\left(k_{F} a\right)^{3 / 2}$-dependence typical for (nearly-) integrable systems.

For the square geometry Eqs. (2.19) and (2.20) for the susceptibilities $\chi^{(\mathrm{t})}$ and $\bar{\chi}$ (characterizing different ensemble averages) can be reduced to [including only the dominant contributions from the family $(1,1)]$ 


$$
\frac{\chi^{(t)}}{\chi^{0}} \simeq \frac{\sqrt{\overline{\chi^{(1)^{2}}}}}{\chi^{0}}=\frac{1}{\sqrt{2}} \int_{0}^{a} \frac{\mathrm{d} x_{0}}{a} \mathscr{C}^{2}\left(x_{0}\right) \cos \left(\varphi \cdot \mathscr{A}\left(x_{0}\right)\right)
$$

and

$$
\frac{\bar{\chi}}{\bar{\chi}^{0}}=\frac{1}{2} \int_{0}^{a} \frac{\mathrm{d} x_{0}}{a} \int_{0}^{a} \frac{\mathrm{d} x_{0}^{\prime}}{a}\left[\mathscr{C}_{-}^{2} \cos \left(\varphi \cdot \mathscr{A}_{-}\right)+\mathscr{b}_{+}^{2} \cos \left(\varphi \cdot \mathscr{A}_{+}\right)\right]
$$

with

$$
\frac{\bar{\chi}^{0}}{\chi_{L}}=\frac{3}{(\sqrt{2} \pi)^{3}}\left(k_{F} a\right) R_{T}^{2}\left(L_{11}\right)
$$

and $\mathscr{b}_{ \pm}=\mathscr{A}\left(x_{0}\right) \pm \mathscr{f}\left(x_{0}^{\prime}\right)$. Although the integrals (2.21), (2.23), and (2.24) can be evaluated analytically in the clean case (leading to Fresnel functions of the magnetic flux ${ }^{5}$ ), the above expressions serve as suitable starting points for the study of disorder effects on ensembles of microstructures discussed in Secs. IV and V.

\section{SEMICLASSICAL APPROACH TO WEAK DISORDER}

Disorder is usually studied in terms of the ensemble average over impurity realizations, since it is a perturbation of an electrostatic potential whose detailed nature is unknown. Typically, quantum perturbation theory is followed by the average over the strengths and positions of the impurities. This approach is suited for macroscopic metallic samples (which are self-averaging) or ensembles of mesoscopic samples (where different samples present different impurity configurations). The possibility of measuring a single disordered mesoscopic sample poses a conceptual difficulty since there is not an average process involved. When discussing the effect of disorder on the orbital magnetism of microstructures, it is therefore necessary to distinguish between the behavior of an individual sample and an ensemble. ${ }^{22}$

Moreover, we have to consider the cases where the Fermi energy and size of the microstructures are kept fixed under impurity average and the cases where these parameters change with the different impurity realizations. These various averages, that will be thoroughly discussed in the remainder of the paper, can be expressed in terms of the impurity average of one- and two-particle Green functions. Therefore we perform in this section a general treatment of disorder effects on the basis of semiclassical expansions of Green functions. The Green function formalism, which is useful for a wide range of physical problems, can be applied to thermodynamical quantities like the magnetic susceptibility (Secs. IV and V) as well as to quantum transport problems.

\section{Disorder models}

Our basic assumptions for the treatment of disorder are the following: We study a spatially random potential $V(\mathbf{r})$ characterized by a correlation function

$$
C\left(\left|\mathbf{r}-\mathbf{r}^{\prime}\right|\right)=\left\langle V(\mathbf{r}) V\left(\mathbf{r}^{\prime}\right)\right\rangle
$$

with a typical correlation length $\xi$ and a mean disorder strength $C^{0}=C(0)$. We will make use of a Gaussian correlation

$$
C\left(\left|\mathbf{r}-\mathbf{r}^{\prime}\right|\right)=C^{0} \exp \left(-\frac{\left(\mathbf{r}-\mathbf{r}^{\prime}\right)^{2}}{4 \xi^{2}}\right)
$$


which allows us to derive analytical expressions for the disorder averages considered below. ${ }^{23}$ The disorder correlation function (3.2) can be viewed as being generated by means of a realization $i$ of a two-dimensional Gaussian disorder potential given by the sum

$$
V(\mathbf{r})=\sum_{j}^{N_{i}} \frac{u_{j}}{2 \pi \xi^{2}} \exp \left\{-\frac{\left(\mathbf{r}-\mathbf{R}_{j}\right)^{2}}{2 \xi^{2}}\right\}
$$

of the potentials of $N_{i}$ independent impurities located at points $\mathbf{R}_{j}$ with uniform probability on an area $\mathrm{V}$. The strengths $u_{j}$ obey $\left\langle u_{j} u_{j^{\prime}}\right\rangle=u^{2} \delta_{j j^{\prime}}$. The disorder strength [as defined in Eq. (3.2)] is

$$
C^{0}=\frac{u^{2} n_{i}}{4 \pi \xi^{2}}
$$

with $n_{i}=N_{i} / \mathrm{V}$. For $\xi \rightarrow 0$ this model yields the white noise case of $\delta$-function scatterers $V(\mathbf{r})=\Sigma_{j}^{N_{i}} u_{j} \delta\left(\mathbf{r}-\mathbf{R}_{j}\right)$. We will use the model of Gaussian disorder for some analytical calculations and for numerical quantum simulations. However, the general results expressed in terms of the correlation function $C\left(\left|\mathbf{r}-\mathbf{r}^{\prime}\right|\right)$ will be valid for any kind of disorder.

As we will show, disorder effects depend on several length scales: the elastic mean free path, the Fermi-wavelength $\lambda_{F}$ of the electrons, the disorder correlation length $\xi$ and the size $a$ of the microstructure. In the bulk case of an unconstrained two-dimensional electron gas (2DEG) we will distinguish between short range $\left(\xi<\lambda_{F}\right)$ and finite range $\left(\xi>\lambda_{F}\right)$ disorder potentials. In the case of a microstructure a third, long range regime for $\xi>a>\lambda_{F}$ has to be considered. The cleanest samples used in today experiments are in the finite range regime $a>\xi>\lambda_{F} \cdot{ }^{24}$

\section{Single-particle Green function}

If we assume a microstructure with size $a \gg \lambda_{F}$ (a condition which is always met in lithographically defined samples) and work in the finite range or long range regime, where the disorder potential is smooth on the scale of $\lambda_{F}$, a semiclassical treatment is well justified. A natural starting point is the semiclassical expression (2.12) for the single-particle Green function $G_{E}\left(\mathbf{r}^{\prime}, \mathbf{r}\right)$ as a sum over the contributions from classical paths. The classical mechanics of trajectories with length $L_{t} \ll l_{T}$ (the transport mean free path) is essentially unaffected by disorder. Therefore the dominant effect on the Green function in Eq. (2.12) results from shifts in the semiclassical phases due to the modification of the actions while the amplitudes $D_{t}$ and topological indices $\eta_{t}$ are nearly unchanged. The first-order approximation to the classical action (2.13) along a path $\mathscr{C}_{t}$ in a system with weak disorder potential is

$$
S_{t}^{d} \simeq S_{t}^{c}+\delta S_{t},
$$

where the clean action $S_{t}^{c}$ is obtained by integrating along the unperturbed trajectory $\mathscr{C}_{t}^{\mathrm{c}}$ without disorder (i.e., $S_{t}^{c}=k_{F} L_{t}$ in the case of billiards without magnetic field) instead of the actual path $\mathscr{C}_{t}$. The correction term $\delta S_{t}$ is obtained, after expanding $\mathbf{p}=\sqrt{2 m[E-V(\mathbf{q})]}$ for small $V / E$, by the integral

$$
\delta S_{t}=-\frac{1}{v_{F}} \int_{\mathscr{C}_{t}^{\mathrm{C}}} V(\mathbf{q}) \mathrm{d} q
$$

In this approximation an impurity average $\langle\ldots\rangle$ acts only on $\delta S_{t}$ and the disorder averaged Green function reads

$$
\left\langle G_{E}\left(\mathbf{r}^{\prime}, \mathbf{r}\right)\right\rangle=\sum_{t} G_{E, t}^{c}\left(\mathbf{r}^{\prime}, \mathbf{r}\right)\left\langle\exp \left[\frac{i}{\hbar} \delta S_{t}\right]\right\rangle .
$$


Here $G_{E, t}^{c}$ is the contribution of the trajectory $t$ to the zero-disorder Green function $G_{E}^{c}$.

For trajectories of length $L_{t} \gg \xi$ the contributions to $\delta S$ according to Eq. (3.6) from the disorder potential at trajectory segments separated by a distance larger than $\xi$ are uncorrelated. The related stochastic accumulation of action along the path can therefore be interpreted as determined by a random-walk process, resulting in a Gaussian distribution of $\delta S_{t}\left(L_{t}\right)$. For larger $\xi$ or shorter trajectories $\left(L_{t} \ngtr \xi\right)$, one can still think of a Gaussian distribution of the de-phasing $\delta S_{t}$ provided $V(\mathbf{r})$ is generated by a sum of a large number of independent impurity potentials. As a consequence of the Gaussian character of the distribution of $\delta S_{t}\left(L_{t}\right)$, the disorder contribution involved in Eq. (3.7) is given by

$$
\left\langle\exp \left[\frac{i}{\hbar} \delta S_{t}\right]\right\rangle=\exp \left[-\frac{\left\langle\delta S_{t}^{2}\right\rangle}{2 \hbar^{2}}\right]
$$

and therefore entirely specified by the variance

$$
\left\langle\delta S_{t}^{2}\right\rangle=\frac{1}{v_{F}^{2}} \int_{\mathscr{C}_{t}^{c}} \mathrm{~d} q \int_{\mathscr{C}_{t}^{c}} \mathrm{~d} q^{\prime}\left\langle V(\mathbf{q}) V\left(\mathbf{q}^{\prime}\right)\right\rangle,
$$

which is expressed as the mean of the disorder correlation function $C\left(\left|\mathbf{q}-\mathbf{q}^{\prime}\right|\right)$ when the unperturbed orbit is traversed.

If we consider, to start with, an unconstrained 2DEG the sum in Eq. (3.7) is reduced to the direct trajectory joining $\mathbf{r}$ and $\mathbf{r}^{\prime}$. If $L=\left|\mathbf{r}-\mathbf{r}^{\prime}\right| \gg \xi$ the inner integral in Eq. (3.9) can be extended to infinity and we obtain

$$
\left\langle\delta S^{2}\right\rangle=\frac{L}{v_{F}^{2}} \int \mathrm{d} q C(\mathbf{q}) .
$$

The semiclassical average Green function for the bulk exhibits therefore an exponential behavior $^{16,25}$ (on a length scale $l_{T}>L \gg \xi$ )

$$
\left\langle G_{E}\left(\mathbf{r}^{\prime}, \mathbf{r}\right)\right\rangle=G_{E}^{c}\left(\mathbf{r}^{\prime}, \mathbf{r}\right) \exp \left(-\frac{L}{2 l}\right),
$$

with the damping governed by an inverse elastic mean free path

$$
\frac{1}{l}=\frac{1}{\hbar^{2} v_{F}^{2}} \int \mathrm{d} q C(\mathbf{q})
$$

In the case of Gaussian correlation $C(\mathbf{q})$ is given by Eq. (3.2) and we get

$$
l=\frac{\hbar^{2} v_{F}^{2}}{\xi \sqrt{\pi} C^{0}} .
$$

Using the disorder strength (3.4) we have

$$
l=\frac{4 \sqrt{\pi} \hbar^{2} v_{F}^{2} \xi}{u^{2} n_{i}} .
$$

In the Appendix we discuss the relation between the semiclassical elastic MFPs [Eqs. (3.12)(3.14)] and the MFP obtained from quantum diagrammatic perturbation theory for the bulk for the disorder model (3.3). The semiclassical and the quantum result [Eq. (A5)] agree asymptotically to 
leading order in $k_{F} \xi$. In the limit of small $\xi$, especially $\xi<\lambda_{F}$, our semiclassical approach is no longer applicable. ${ }^{26}$ However, Eq. (3.11) still holds, but with $l$ replaced by $l_{\delta}$ given in Eq. (A4).

We now turn from the semiclassical treatment of the bulk to that of a confined system. In the constrained case in the limit $l_{T} \ll a$ impurity scattering is the dominant process. ${ }^{27}$ This gives rise to diffusive motion, and thus there is no essential difference to the bulk for the damping of the Green function. We will treat the ballistic regime $l_{T}>a$ where both, the confinement and the impurities have to be considered. The calculation of $l_{T}$ in the Appendix shows that for finite $\xi$ the transport MFP $l$ is considerably larger than the elastic one and a ballistic treatment is therefore well justified, even if $l$ is of the order of the system size.

In contrast to the bulk case a disorder averaged confined system is no longer translationally invariant and one has to impose in quantum calculations the correct boundary conditions of the geometry. Confinement implies semi-classically that $G_{E}^{c}\left(\mathbf{r}^{\prime}, \mathbf{r}\right)$ is given as a sum over all direct and multiply reflected paths connecting $\mathbf{r}$ and $\mathbf{r}^{\prime}$; disorder modifies the corresponding actions according to Eq. (3.6).

In the regimes of short- and finite-range scatterers, the damping of each contribution $\left\langle G_{E, t}\right\rangle$ to $\left\langle G_{E}\right\rangle$ is given, analogous to the bulk expression (3.11), [using Eq. (3.10)] by

$$
\left\langle G_{E}\left(\mathbf{r}^{\prime}, \mathbf{r}\right)\right\rangle=\sum_{t} G_{E, t}^{c}\left(\mathbf{r}^{\prime}, \mathbf{r}\right) \exp \left(-\frac{L_{t}}{2 l}\right) .
$$

Here, $L$ is now replaced by the trajectory length $L_{t}>a \gg \xi$. This gives an individual damping $\exp \left(-L_{t} / 2 l\right)$ for each geometry-affected path contributing to $\left\langle G_{E}\right\rangle$.

In the long range regime and for $\xi \sim a$ the correlation integral (3.9) can no longer be approximated (as for $\xi \ll L_{t}$ ) by $L \int_{-\infty}^{+\infty} d q C(\mathbf{q})$ due to correlations across different sectors of an orbit (with distance smaller $\xi$ ). Therefore, the orbit-geometry enters into the correlation integral. For $\xi \gg a$ we can, however, expand $C\left(\left|\mathbf{r}-\mathbf{r}^{\prime}\right|\right)$ and obtain in the case of Gaussian disorder (up to first order in $\left.\xi^{-2}\right) C\left(\left|\mathbf{r}-\mathbf{r}^{\prime}\right|\right) \simeq C^{0}\left[1-\left(\mathbf{r}-\mathbf{r}^{\prime}\right)^{2} /\left(4 \xi^{2}\right)\right]$. In this approximation the integral (3.9) gives for the Green function damping an exponent

$$
\frac{\left\langle\delta S_{t}^{2}\right\rangle}{2 \hbar^{2}}=\frac{1}{4 \sqrt{\pi}} \frac{L_{t}^{2}}{l \xi}\left(1-\frac{1}{2} \frac{I_{t}}{\xi^{2}}\right) .
$$

$I_{t}=\left(1 / L_{t}\right) \int_{\mathscr{C}_{t}} \mathbf{r}^{2}(q) \mathrm{d} q$ can be regarded as the "moment of inertia" of the unperturbed trajectory $\mathscr{C}_{t}$ with respect to its "center of mass" $\left(1 / L_{t}\right) \int_{\mathscr{C}_{t}} \mathbf{r}(q) \mathrm{d} q$. Eq. (3.16) shows that the damping in the long range regime depends quadratically on $L_{t}$ (in contrast to linear behavior in the finite range case or bulk). The length scale of damping is now given by the geometrical mean of the bulk MFP $l$ and $\xi$. The leading damping term does not depend on the specific orbit geometry since it essentially reflects the fluctuation in the mean of the (smooth) potentials of different impurity configurations. Inclusion of higher powers of $\xi^{-2}$ leads to additional contributions from higher moments $\int_{\mathscr{C}_{t}} r^{n}(q) \mathrm{d} q$ on the RHS of Eq. (3.16).

\section{Two-particle Green function}

Density correlation functions in general or the typical [Eq. (2.19)] and ensemble averaged susceptibility [Eq. (2.20)], which will be treated in the subsequent sections, involve the square of the density of states. Writing the latter, Eq. (2.11), in terms of the difference between advanced and retarded Green functions $\left(G^{+}-G^{-}\right)$we are left with products of one-particle Green functions. The terms of most interest are the cross products $G^{+}\left(r, r^{\prime}\right)$ $\times G^{-}\left(r, r^{\prime}\right)=G^{+}\left(r, r^{\prime}\right) G^{+*}\left(r^{\prime}, r\right)$, because they survive the energy average and are sensitive to changes in the magnetic field. 
Since, in the non-interacting approach we are using, the two-particle Green function factorizes into a product of one-particle Green function ${ }^{28}$ we will use the former as a synonym for the latter. The semiclassical average for products of single-particle Green functions will be quantitatively performed for the susceptibility of confined integrable systems in Sec. V, and we discuss here the underlying ideas for the general case.

Considering for instance the product $G\left(r_{1}, r_{2}\right) G^{*}\left(r_{1}^{\prime}, r_{2}^{\prime}\right)$, the effect of the disorder potential can be taken into account perturbatively for each realization of the disorder in the same way as before by Eqs. (3.5)-(3.6). Using the same kind of argument, one can therefore write the disorder average as a double sum over the averaged contributions from trajectories $t$ and $t^{\prime}$

$$
\begin{aligned}
\left\langle G_{E} G_{E}^{*}\right\rangle & =\sum_{t} \sum_{t^{\prime}}\left\langle G_{E, t} G_{E, t^{\prime}}^{*}\right\rangle=\sum_{t} \sum_{t^{\prime}} G_{E, t}^{c} G_{E, t^{\prime}}^{c *}\left\langle e^{(i / \hbar)\left(\delta S_{t}-\delta S_{t^{\prime}}\right)}\right\rangle \\
& =\sum_{t} \sum_{t^{\prime}} G_{E, t}^{c} G_{E, t^{\prime}}^{c^{*}} \exp \left[-\frac{\left\langle\left(\delta S_{t}-\delta S_{t^{\prime}}\right)^{2}\right\rangle}{2 \hbar^{2}}\right] .
\end{aligned}
$$

It is necessary here, however, to take into account the correlation of the disorder potential between points on trajectories $t$ and $t^{\prime}$. One limiting case for instance would be that $t$ and $t^{\prime}$ are either the same trajectory or the time reversal one of each other. In these cases their contribution acquires exactly the same phase shift and $\left\langle G_{E, t} G_{E, t}^{*}\right\rangle=\left|G_{E, t}^{c}\right|^{2}$. Within our approximation the diagonal contributions $t=t^{\prime}$, which, e.g., are responsible for the classical part of the conductivity, remain thus disorder-unaffected, since we assume the trajectories have a length much smaller than $l_{T}$. (A semiclassical consideration of these effects for trajectories of length of the order of $l_{T}$ or larger was performed in Ref. 25 for the bulk, giving a damping of the two-point Green function on the scale of $l_{T}$.) At the opposite extreme, if trajectories $t, t^{\prime}$ are completely uncorrelated, i.e., for long trajectories in classical chaotic systems or trajectories in integrable systems with a spatial distance larger than $\xi$, the average in Eq. (3.17) factorizes: $\left\langle G_{E, t} G_{E, t^{\prime}}^{*}\right\rangle=\left\langle G_{E, t}\right\rangle \cdot\left\langle G_{E, t^{\prime}}^{*}\right\rangle$ and lead to singleparticle damping behavior.

The double sum Eq. (3.17) may however involve pairs of trajectories which stay within a distance of the order of $\xi$ (as for nearby paths on a torus of an integrable system). In this case the behavior of $\left\langle G_{E, t} G_{E, t^{\prime}}^{*}\right\rangle$ is more complicated and depends of the confinement geometry of the system under consideration. As a simple illustration of the interplay between disorder correlation and families of orbits, let us consider for the case of the bulk the product of $G\left(r_{1}, r_{2}\right)$ joining $r_{1}=(0,0)$ to $r_{2}=(L, 0)$ with $G^{*}\left(r_{1}^{\prime}, r_{2}^{\prime}\right)$ joining $r_{1}=(0, y)$ to $r_{2}=(L, y)$, with $L \gg \xi$ but $y$ possibly of the order of $\xi$. Introducing the function

$$
K(y)=\int_{-\infty}^{+\infty} C(x, y) \mathrm{d} x
$$

[for Gaussian correlations Eq. (3.2), $K(y) / K(0)=\exp \left(-y^{2} /\left(4 \xi^{2}\right)\right)$ ], the variance of the de-phasing is obtained as

$$
\left\langle\left(\delta S_{t}-\delta S_{t^{\prime}}\right)^{2}\right\rangle=2 L \frac{(K(0)-K(y))}{v_{F}^{2}}
$$

and therefore $\left\langle G_{E} G_{E}^{*}\right\rangle=G_{E}^{c} G_{E}^{c *} \widetilde{f}(y)$ with

$$
\widetilde{f}(y)=\exp \left[-\frac{L}{l}\left(1-\frac{K(y)}{K(0)}\right)\right]
$$


The function $\widetilde{f}(y)$ expresses in a very simple way that as $y \rightarrow 0$, the effect of disorder disappears $(\widetilde{f}(0)=1)$ while for $y \gg \xi$ the function $\widetilde{f}(y)$ behaves as the square of single particle Green function damping.

\section{FIXED-SIZE IMPURITY AVERAGE OF THE MAGNETIC SUSCEPTIBILITY}

We consider here a disorder average (which will henceforth be called a fixed-size impurity average) of an ensemble of structures for which the parameters of the corresponding clean system (geometry, size, chemical potential) remain fixed under the change of impurity realizations. In Sec. V, we will then treat the more realistic case of the orbital magnetic response of a combined energy (or size) and disorder average.

As shown in the previous section, averages over weak disorder exponentially damp, but do not completely suppress oscillatory contributions (with phase $k_{F} L_{t}$ ) to the single-particle Green function from geometrical paths in confined systems. An observable quantity dependent on these contributions is the disorder averaged susceptibility of an ensemble of billiards of the same size or same clean-system Fermi energy, which will be studied first.

We will treat regular billiards at zero or small magnetic fields, where the integrability is approximately maintained and the density of states has the $H$-dependence of the formulae (2.14)(2.16). The general result for $\chi^{(1)}$, Eq. (2.17), formally persists with the replacement of $\mathscr{C}_{\mathrm{M}}$ by

$$
\left\langle\mathscr{C}_{\mathbf{M}}(H)\right\rangle=\frac{1}{2 \pi} \int_{0}^{2 \pi} \mathrm{d} \Theta_{1} \cos \left[2 \pi \frac{H \mathscr{C}_{\mathbf{M}}\left(\Theta_{1}\right)}{\Phi_{0}}\right] \exp \left[-\frac{\left\langle\left(\delta S_{\mathrm{M}}\left(\Theta_{1}\right)\right)^{2}\right\rangle}{2 \hbar^{2}}\right],
$$

where $\left\langle\delta S_{\mathrm{M}}^{2}\left(\Theta_{1}\right)\right\rangle$ is given by Eq. (3.9) with the integrals performed along the orbits of the family $\mathbf{M}$ parameterized by $\Theta_{1}$. In the finite range case (if all orbits of a family $\mathbf{M}$ are of the same length as in billiards) each family exhibits a unique disorder damping giving a contribution

$$
\left\langle\chi_{\mathrm{M}}^{(1)}\right\rangle=\chi_{\mathrm{M}}^{(1)} \cdot \exp \left(-\frac{\left\langle\delta S_{\mathrm{M}}^{2}\right\rangle}{2 \hbar^{2}}\right)
$$

to the ballistic susceptibility. $\chi_{\mathbf{M}}^{(1)}$ is the contribution of family $\mathbf{M}$ to the clean susceptibility [Eq. (2.17)] and $\left\langle\delta S_{\mathrm{M}}^{2}\right\rangle / 2 \hbar^{2}=L_{\mathrm{M}} / 2 l$.

In the case of square billiards, where the dominant contribution stems from the family $(1,1)$, we obtain, in analogy with Eq. (2.21),

$$
\frac{\langle\chi\rangle}{\chi^{0}} \simeq \frac{\left\langle\chi^{(1)}\right\rangle}{\chi^{0}}=\int_{0}^{a} \frac{\mathrm{d} x_{0}}{a} \mathscr{b}^{2}\left(x_{0}\right) \cos \left(\varphi \mathscr{C}\left(x_{0}\right)\right)\left\langle\sin \left(k_{F} L_{11}+\frac{\pi}{4}+\frac{\delta S\left(x_{0}\right)}{\hbar}\right)\right\rangle
$$

with $\chi^{0}$ given by Eq. (2.22). For a square billiard $\delta S\left(x_{0}\right)$ is independent of $x_{0}$ for the finite- as well as for the long-range regime since $I_{11}=a^{2} / 12$ [entering into Eq. (3.16)] is the same for all orbits (11). Therefore Eq. (4.2) with $\mathbf{M}=(1,1)$ holds for both limiting cases. In the same way as for the damping of the one-particle Green function [Eq. (3.15)] we obtain for square billiards at finite temperature in the finite range regime

$$
\langle\chi\rangle \simeq\left\langle\chi^{(1)}\right\rangle=\chi_{\mathrm{cl}}^{(1)} \cdot \exp \left(-\frac{L_{11}}{2 l}\right),
$$

where $\chi_{\mathrm{cl}}^{(1)}$ denotes the susceptibility of the system without disorder.

In order to control the validity of our analytical semiclassical approximations we performed numerical quantum calculations by diagonalizing the Hamiltonian for non-interacting particles in a square billiard subject to a uniform perpendicular magnetic field and a random disorder potential of the form of Eq. (3.3). For a given selected correlation length $\xi$ a quantum mechanically 
calculated elastic MFP $l_{\mathrm{qm}}$ and a fixed Fermi momentum $k_{F}$ the product of the impurity density and squared mean impurity potential, $n_{i} u^{2}$, is determined by Eqs. (A3) and (A4). We found that our numerical results are essentially independent of the choice of $n_{i}$ (with $u^{2}$ adjusted accordingly) for $n_{i} \geqslant 200$ and used this value for the calculations presented here. The positions $\mathbf{R}_{j}$ of the impurities were chosen as independently distributed and for the $u_{j}$ we used a box distribution.

Each impurity configuration $\alpha$ has a self-averaging effect for an individual square billiard (for $\xi<a)$ due to the differences of the impurity potential $V_{\alpha}(\mathbf{r})$ across the structure. In an average over an ensemble of square billiards, differences in the mean impurity potential $\overline{V_{\alpha}}=\left(1 / a^{2}\right) \int \mathrm{d} \mathbf{r} V_{\alpha}(\mathbf{r})$ (the integral is taken over the area of the billiard) between different squares lead to an additional damping. It is characterized by the variance

$$
\begin{aligned}
& \left\langle\bar{V}^{2}\right\rangle=\frac{u^{2} n_{i}}{a^{2} \eta^{2}}\left[\eta \operatorname{erf}(\eta)+\frac{1}{\sqrt{\pi}}\left(e^{-\eta^{2}}-1\right)\right]^{2} ; \quad \eta=\frac{a}{2 \xi} \\
& \rightarrow \frac{u^{2} n_{i}}{4 \pi \xi^{2}} \quad \text { for } \xi / a \rightarrow \infty \\
& \rightarrow \frac{u^{2} n_{i}}{a^{2}} \text { for } \xi \rightarrow 0
\end{aligned}
$$

In the limit of $\xi \gg a$ our numerical calculations showed that the self-averaging effect is negligible (since the impurity potential is essentially flat across the square) and the clean susceptibility of an individual structure remains practically unaffected by disorder. In this limit variations in the mean potential $\bar{V}$ of an ensemble [Eq. (4.6)] dominate the damping. In the limit of short range disorder, fluctuations in the mean $\bar{V}$ of different samples play a minor role and self-averaging is the predominant process for an integrable system: In semi-classical terms different trajectories of a family of closed orbits are perturbed by white noise disorder in an uncorrelated manner. Therefore we do not observe considerable differences between the susceptibility of a single disordered billiard of integrable geometry and the corresponding ensemble for $\xi \ll a$. In a chaotic billiard this self-averaging effect does not exist (for not too small $\xi$, see end of Sec. V), since orbits are isolated. Therefore distinct differences between an individual disordered sample and an ensemble of disordered billiards are expected.

To improve the statistics of our numerical ensemble average for square billiards we performed an average over disorder configurations with the same mean $\bar{V}$ and in addition averaged over $\bar{V}$ according to Eq. (4.5) ${ }^{29}$ Fig. 2 shows results of the numerical quantum simulations for the average susceptibility $\langle\chi\rangle$ of an ensemble of squares with fixed size but different disorder realizations at a temperature $k_{B} T=3 \mathrm{~g}_{\mathrm{s}} \Delta$, where $\Delta$ is the mean level spacing. The characteristic oscillations in $k_{F} a$ show an interchange between para- and dia-magnetic behavior on a scale $k_{F} L_{11}$. This indicates that they are dominated by contributions from the shortest flux-enclosing orbits of the family $(1,1)$ [according to Eqs. (2.17) and (4.3)], as has been already shown for the clean case in Refs. 5 and 6. Fig. 2 demonstrates the damping of the clean susceptibility (dotted line) with decreasing elastic MFP $l / a=4,2,1,0.5$ for fixed $\xi / a=0.1$ (which represents a typical disorder correlation length in experimental realizations). Variations in the mean $\bar{V}$ lead to a de-phasing of the oscillations in the finite range case on a scale $(\delta k) a \sim(4 \pi)^{1 / 4} \sqrt{\xi / l_{\mathrm{qm}}(\xi)}$ which is, as discussed above, small compared to the self-averaging effect in this regime.

Figure 3 depicts the quantitative comparison between numerical and analytical results: It shows the logarithm of $\langle\chi\rangle$ normalized to the corresponding zero-disorder susceptibility as a function of the inverse MFP for different correlation lengths $\xi$. The semi-classically predicted exponential damping [Eq. (4.2)] is shown as straight lines for the short range $[\xi \ll a$, Eq. (3.11), 


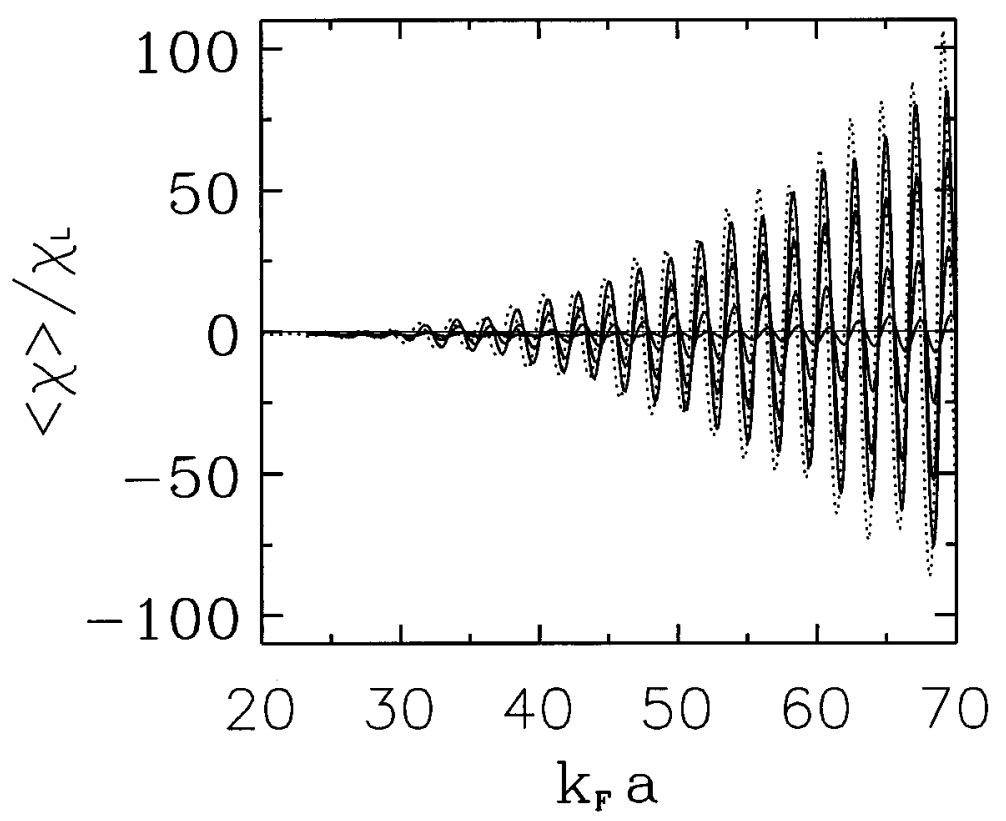

FIG. 2. Magnetic susceptibility $\langle\chi\rangle$ (normalized with respect to the Landau susceptibility $\chi_{L}$ ) of a square billiard as a function of $k_{F} a$ for the clean case (dotted) and for the ensemble average of billiards of fixed size with increasing Gaussian disorder $(\xi / a=0.1)$ according to an elastic mean free-path $l / a=4,2,1,0.5$ (solid lines in the order of decreasing amplitude). The susceptibility is calculated for zero magnetic field and at a temperature equal to 6 level spacings.

full line for $\xi=0]$ and long range [ $\xi>a$, Eq. (3.16), dotted lines for $\xi / a=4,2,1$ from the top]. The semiclassical predictions accurately agree with the corresponding quantum results (symbols) for $\xi / a=4,2,1,0$ and fail for intermediate values $\xi / a=0.5,0.2$ (squares and diamonds) which are off the range of validity of the approximations. The transition from self-averaging dominated $(\xi \rightarrow 0)$ suppression to damping according to fluctuations in the floor $\bar{V}$ (for $\xi / a \rightarrow \infty)$ turns out to be non-monotonic.

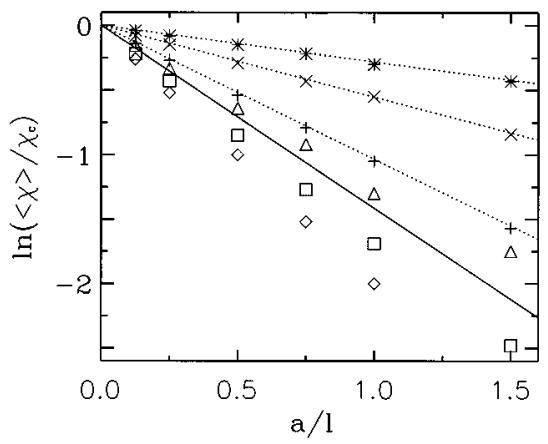

FIG. 3. Logarithm of the ratio $\langle\chi\rangle / \chi_{c l}$ as a function of the inverse elastic MFP $a / l$. The symbols indicate the numerical quantum results (from the top for $\xi / a=4,2,1,0,0.5$ and 0.2 . The dotted lines show the semiclassical analytical results for $\xi / a=4,2,1$ (from above) according to Eq. (3.16). The full line is the semiclassical result for $\xi=0$ [Eq. (3.11)]. The quantum results for $\xi=0.5$ (squares) and 0.2 (diamonds) are beyond the regime of validity of the analytical limits $\xi / a \gg 1$ and $\xi / a \ll 1$. 


\section{COMBINED IMPURITY- AND ENERGY-AVERAGE OF THE SUSCEPTIBILITY}

In currently experimentally realizable structures disorder averages cannot be performed independently from size-averages since the detailed features of the confining potential do not remain unchanged for different impurity configurations. From the basic expressions (2.21) and (4.3) for the susceptibility we see that changes in size $a$ give rise to rapid variations in the phase $k_{F} a$ (on a quantum scale) and a much slower secular variation through the geometrical factors $A$. Thus, the effect of small size variations is equivalent to an energy $\left(k_{F}\right)$ average. As discussed in Sec. II for the clean case, variations in $k_{F}$ lead to vanishing $\chi^{(1)}$. Therefore we have to use the typical and energy averaged susceptibilities [see Eqs. (2.19) and (2.20) for their definition in the clean case]. When disorder is introduced we must consider energy- and disorder averages. The typical susceptibility is now defined by $\chi^{(t)}=\left\langle\overline{\chi^{2}}\right\rangle^{1 / 2}$. It applies to the case of repeated measurements on a given microstructure when different impurity realizations (and simultaneous changes in $k_{F}$ ) are obtained by some kind of perturbation (e.g., cycling to room temperature). From now on we will reserve the term $\chi_{\mathrm{cl}}^{(t)}$ for the clean typical susceptibility $\left(\overline{\chi^{2}}\right)^{1 / 2}$. The energy and impurity averaged susceptibility $\langle\bar{\chi}\rangle$ describes the magnetic response of an ensemble of a large number of microstructures with different impurity realizations and variations in size. This is the situation of the experiment of Ref. 21 that we discuss in the sequel.

\section{A. Integrable systems: The square billiard}

The semiclassical results for $\chi^{(t)}$ and $\langle\vec{\chi}\rangle$ for a system of integrable geometry are obtained in an analogous way as we proceeded for $\langle\chi\rangle$ in Sec. IV, that is by including in the integral (2.16) for $\mathscr{C}_{\mathbf{M}}$ a $\Theta_{1}$-dependent disorder-induced phase $\exp \left(i \delta S\left(\Theta_{1}\right) / \hbar\right)$ [see Eq. (4.1)]. However, now we have to take the square of $\mathscr{C}_{\mathbf{M}}$ (respectively, $\partial^{2} \mathscr{C}_{\mathbf{M}} / \partial H^{2}$ ) before the impurity average and cross correlations between different paths $\Theta$ and $\Theta^{\prime}$ on a torus $\mathbf{M}$ or between different tori have to be considered. We discuss this effect, typical of integrable systems, for the case of a square billiard. For sake of clarity we assume moreover a temperature range such that only the contribution of the shortest closed orbit has to be taken into account. Instead of Eqs. (2.23) and (2.24) which hold for the clean case, the contribution of orbits of topology $\mathbf{M}=(1,1)$ for the typical susceptibility now reads

$$
\left(\frac{\chi^{(t)}}{\chi^{0}}\right)^{2}=\frac{1}{2} \int_{0}^{a} \frac{\mathrm{d} x_{0}}{a} \int_{0}^{a} \frac{\mathrm{d} x_{0}^{\prime}}{a} \mathscr{C}^{2}\left(x_{0}\right) \mathscr{C}^{2}\left(x_{0}^{\prime}\right) \cos \left(\varphi \mathscr{C}\left(x_{0}\right)\right) \cos \left(\varphi \mathscr{C}\left(x_{0}^{\prime}\right)\right) f\left(x_{0}, x_{0}^{\prime}\right),
$$

with $\chi^{0}$ defined as in Eq. (2.22). The function

$$
\begin{aligned}
f\left(x_{0}, x_{0}^{\prime}\right) & =\left\langle\exp \left\{\frac{i}{\hbar}\left(\delta S\left(x_{0}\right)-\delta S\left(x_{0}^{\prime}\right)\right)\right\}\right\rangle \\
& =\exp \left\{-\frac{1}{2 \hbar^{2}}\left[\left\langle\delta S^{2}\left(x_{0}\right)\right\rangle+\left\langle\delta S^{2}\left(x_{0}^{\prime}\right)\right\rangle-2\left\langle\delta S\left(x_{0}\right) \delta S\left(x_{0}^{\prime}\right)\right\rangle\right]\right\}
\end{aligned}
$$

accounts for the effect of disorder on pairs of orbits $x_{0}$ and $x_{0}^{\prime}$. [See Eq. (3.20) for the treatment in the general case]. For the magnetic response of an energy- and disorder-averaged ensemble we find correspondingly

$$
\frac{\langle\bar{\chi}\rangle}{\bar{\chi}}=\frac{1}{2} \int_{0}^{a} \frac{\mathrm{d} x_{0}}{a} \int_{0}^{a} \frac{\mathrm{d} x_{0}^{\prime}}{a}\left[\mathscr{C}_{-}^{2} \cos \left(\varphi \cdot \mathscr{C}_{-}\right)+\mathscr{A}_{+}^{2} \cos \left(\varphi \cdot \mathscr{C}_{+}\right)\right] f\left(x_{0}, x_{0}^{\prime}\right)
$$

with $\bar{\chi}^{0}$ defined in Eq. (2.25) and $\mathscr{C}_{ \pm}$as in Eq. (2.24). 


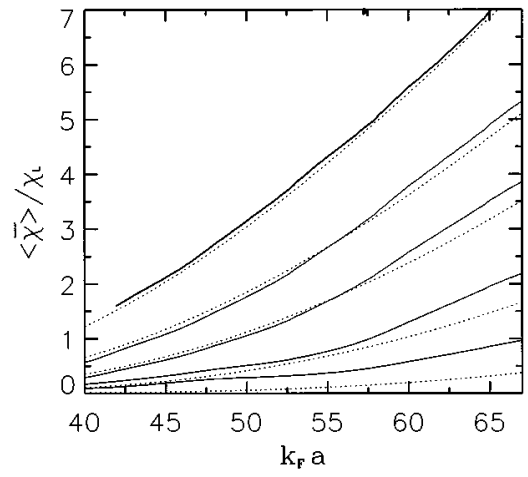

FIG. 4. Averaged magnetic susceptibility (at $H \approx 0$ of an ensemble of square billiards with variations in the size and impurity potential $(\xi=0)$ for different disorder strength, i.e., elastic mean free path $l_{\delta}$. The full curves show the numerical quantum results and the dotted lines the semiclassical predictions from Eq. (5.6) taking into account the variations of $l_{\delta}$ with $k_{F}$ [see Eq. (A4)]. The two sets of curves correspond to an elastic MFP $l_{\delta} / a=\infty, 8,4,2,1$ (at $k_{F} a=65$ ), (from the top).

\section{Short range case}

We begin with the discussion of the short range case: Although we reach the border of applicability of our semiclassical approximation for $\xi \rightarrow 0$, it shows us that in this limit orbits with $x_{0} \neq x_{0}^{\prime}$ are disorder-uncorrelated and all such pair contributions are exponentially damped. Using exclusively the family $(1,1)$, one obtains an overall suppression of the typical and average susceptibility at finite temperature according to

$$
\begin{gathered}
\lim _{\xi \rightarrow 0} \chi^{(t)}=\chi_{\mathrm{cl}}^{(t)} e^{-L_{11} / 2 l_{\delta},} \\
\lim _{\xi \rightarrow 0}\langle\bar{\chi}\rangle=\bar{\chi} e^{-L_{11} / l_{\delta}} .
\end{gathered}
$$

Note that the exponent for $\langle\bar{\chi}\rangle$ differs by a factor $1 / 2$ from that for $\langle\chi\rangle$ [see Eq. (4.2) and subsequent text].

Figure 4 depicts the $k_{F} a$ dependence of the ensemble averaged susceptibility $\langle\bar{\chi}\rangle$ in the short range case $\xi=0$. The dotted curves showing the semiclassical analytical formula (5.6) are compared with a direct quantum mechanical calculation of $\left\langle\chi^{(2)}\right\rangle$ [using the numerically obtained $N^{\text {osc }}(\bar{\mu})$ in Eq. (2.9c)] for disorder ensembles of different impurity strength equivalent to an elastic MFP $l_{\delta} / a=\infty, 8,4$, and 1 at $k_{F} a \sim 65$ (from the top). Note, that the effective MFP decreases along the curves with decreasing $k_{F}$ [see Eq. (A4)] and the localized regime may eventually be reached for small $k_{F} a$. At the limit of the ballistic regime at small $l \sim a$ the semiclassical result begins to differ from the quantum one although the functional behavior remains the same. This arising difference may be related to non-ballistic scattering from impurities which is not included here.

\section{Finite range case}

In the finite range $\lambda_{F}<\xi \ll a$, the phase shifts $\delta S\left(x_{0}\right)$ and $\delta S\left(x_{0}^{\prime}\right)$ in $f\left(x_{0}, x_{0}^{\prime}\right)$ are accumulated in a correlated way, if the spatial distance of two orbits $x_{0}$ and $x_{0}^{\prime}$ is smaller than $\xi$. To evaluate the product term $2\left\langle\delta S\left(x_{0}\right) \delta S\left(x_{0}^{\prime}\right)\right\rangle$ in the exponent of $f\left(x_{0}, x_{0}^{\prime}\right)$ in this regime the integrations are performed as in Eq. (3.9) but with $\mathbf{q}$ and $\mathbf{q}^{\prime}$ running along paths starting at $x_{0}$, respectively, $x_{0}^{\prime}$. Ignoring the additional correlations occuring near the bounces off the boundaries of the billiard, the trajectories $x_{0}$ and $x_{0}^{\prime}$ (see Fig. 1) can be regarded as straight lines remaining at a 
constant distance $y=\left|x_{0}-x_{0}^{\prime}\right| / \sqrt{2}$ from another. We can therefore approximate $f\left(x_{0}, x_{0}^{\prime}\right)$ by $\widetilde{f}\left(\left|x_{0}-x_{0}^{\prime}\right| / \sqrt{2}\right)$ with the function $\widetilde{f}$ given by Eq. (3.20). For Gaussian correlation we thus have

$$
f\left(x_{0}, x_{0}^{\prime}\right)=\exp \left\{-\frac{L_{11}}{l}\left[1-\exp \left(-\frac{\left(x_{0}-x_{0}^{\prime}\right)^{2}}{8 \xi^{2}}\right)\right]\right\} \text {. }
$$

Orbits separated by $\left|x_{0}-x_{0}^{\prime}\right| \gg \xi$ are disorder-uncorrelated and exponentially suppressed: $f\left(x_{0}, x_{0}^{\prime}\right) \simeq \exp \left(-L_{11} / l\right)$. For those orbits the random disorder leads to an uncorrelated detuning of the phases. In contrast to that, disorder only weakly affects trajectories separated by $\left|x_{0}-x_{0}^{\prime}\right|<\xi$.

The disorder averages in the finite range regime lead, by means of the function $f$, to a non-exponential damping of the susceptibilities for systems with families of periodic orbits. This behavior becomes obvious for the case of square billiards where at $H=0$ the integrals (5.1) and (5.4) can be evaluated analytically in the limits of $L_{11} \ll l$ (extreme ballistic) and $L_{11} \gg l$ (deep ballistic). We find for the typical and average susceptibility at $H=0$ in the finite range case for $L_{11} \ll l$

$$
\begin{gathered}
\left(\frac{\chi^{(t)}}{\chi_{\mathrm{cl}}^{(t)}}\right)^{2} \simeq 1-\frac{L_{11}}{l}\left(1-c_{t} \frac{\xi}{a}\right), \\
\frac{\langle\bar{\chi}\rangle}{\bar{\chi}} \simeq 1-\frac{L_{11}}{l}\left(1-c_{a} \frac{\xi}{a}\right),
\end{gathered}
$$

and for $L_{11} \gg l$ (by steepest descent)

$$
\begin{gathered}
\left(\frac{\chi^{(t)}}{\chi_{\mathrm{cl}}^{(t)}}\right)^{2} \simeq c_{t}\left(\frac{\xi}{a}\right)\left(\frac{l}{L_{11}}\right)^{1 / 2}, \\
\frac{\langle\bar{\chi}\rangle}{\bar{\chi}} \simeq c_{a}\left(\frac{\xi}{a}\right)\left(\frac{l}{L_{11}}\right)^{1 / 2} .
\end{gathered}
$$

The constants in the above equations are $c_{t}=(20 / 7) \sqrt{2 \pi}$ and $c_{a}=2 \sqrt{2 \pi}$. Eqs. (5.8) express the limit of very weak disorder, showing that the small disorder effect is further reduced due to the correlation of the disorder potential. The other limit, Eqs. (5.9), is noticeably more interesting since it shows that disorder correlation effects lead to a replacement of the exponential disorder damping by a power law.

Figure 5 depicts in logarithmic representation our collected results for the disorder averaged typical (a) and averaged (b) susceptibility for square billiards (at $H=0$ and $k_{B} T=2 \mathrm{~g}_{\mathrm{s}} \Delta$ ) as a function of the inverse elastic MFP for different disorder correlation lengths. The symbols denote results from numerical quantum simulations described in the previous section and the full curves semiclassical results from numerical integration of the Eqs. (5.1) and (5.4). For the short range case $\xi=0$ they reduce to Eq. (5.6) predicting an exponential decrease with exponent $L_{11} / l$ which is in line with the quantum calculations (circles). The semiclassical results for the finite range are on the whole in agreement with the numerical results for $\xi / a=0.1$ (diamonds), $\xi / a=0.2$ (triangles) and $\xi / a=0.5$ (squares). The semiclassical curves seem to overestimate the damping of the typical susceptibility. The dotted curves (shown for $a / l \geqslant 1$ ) depict the analytical expressions (5.9) in the regime $L_{11}>l$. Since for finite $\xi$ the transport MFP $l_{T}>l$ [see Eq. (A6)], this regime can still be considered as (deep) ballistic and our semiclassical assumptions being based on straight-line trajectories remain valid. 

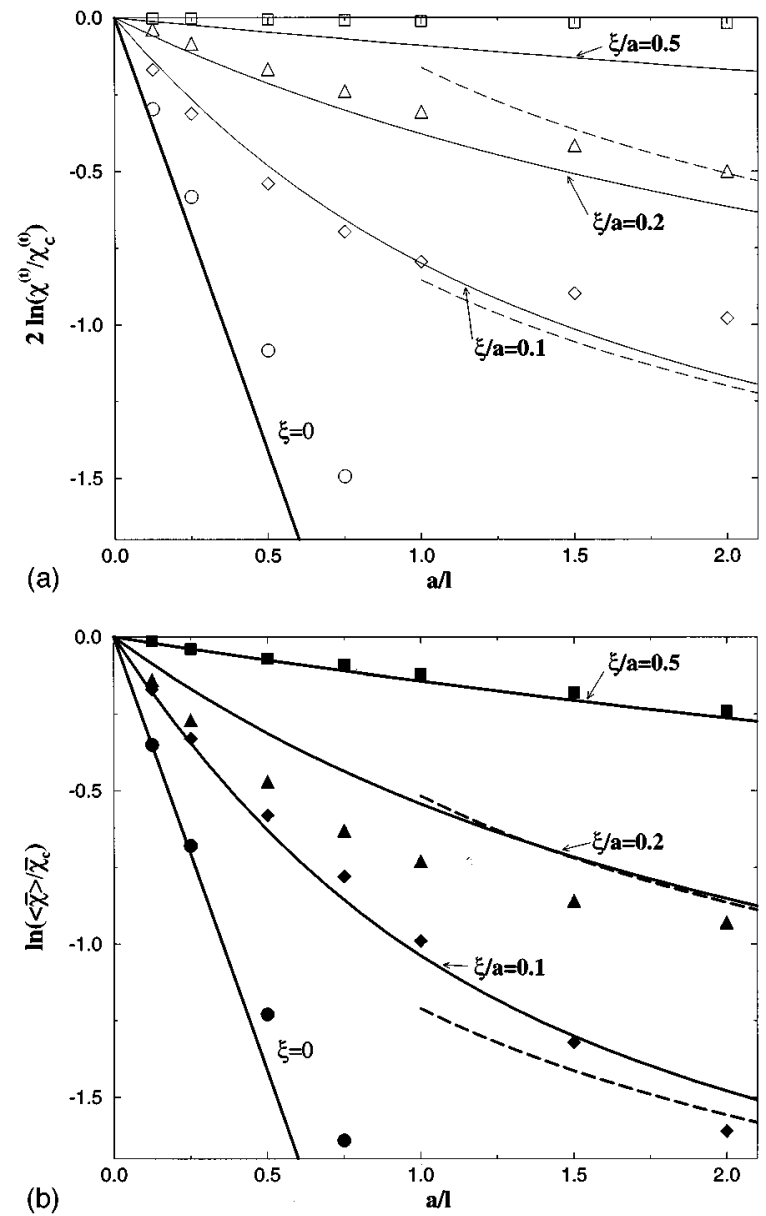

FIG. 5. Logarithm of the ratio between disorder averaged and clean results for (a) typical $\chi^{(t)}$ (b) ensemble averaged $\langle\bar{\chi}\rangle$ susceptibilities as a function of increasing inverse elastic MFP $a / l$ for different values of $\xi / a$. The symbols denote the numerical quantum results, the solid lines (for $\xi>0$ ) the semiclassical integrals (5.1) (a) and (5.4) (b) and the dashed lines asymptotic expansions (5.9) of the integrals for large $a / l$.

As the semiclassical formulae already indicate, the overall disorder behavior of $\langle\bar{\chi}\rangle$ and $\chi^{(t)}$ is quite similar.

\section{Long range case}

For completeness, we will consider the effect of the disorder for the long range regime: $\mathrm{We}$ can use the Eqs. (5.1) and (5.4) but cannot calculate the disorder function $f\left(x_{0}, x_{0}^{\prime}\right)$ in the same way as for the finite range. We can however, similar as for $\langle\chi\rangle$ in Sec. IV, expand the exponent $-\left\langle\left(\delta S\left(x_{0}\right)-\delta S\left(x_{0}^{\prime}\right)\right)^{2}\right\rangle$ of $f\left(x_{0}, x_{0}^{\prime}\right)$ in Eq. (5.2) for small $a / \xi$. In the case of the square all orders up to $(a / \xi)^{8}$ vanish and we find a very small overall reduction of the clean averaged susceptibilities [from family (11)] given by

$$
\left(\frac{\chi^{(t)}}{\chi_{\mathrm{cl}}^{(t)}}\right)^{2} \simeq 1-6.5 \cdot 10^{-5} \frac{a}{l}\left(\frac{a}{\xi}\right)^{9}
$$

For square billiards this leading order contribution no longer depends on $x_{0}$. The energy- and disorder-average $\langle\bar{\chi}\rangle$ exhibits the same damping as $\left(\chi^{(t)}\right)^{2}$. Note that besides the high order in 


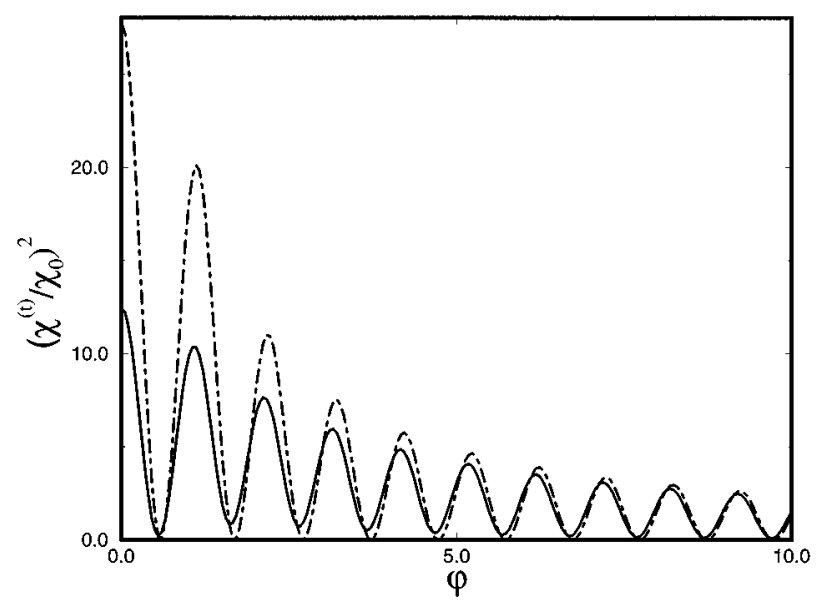

FIG. 6. Typical susceptibility as predicted by Eq. (5.1) as a function of the dimensionless flux $\varphi=H a^{2} / \Phi_{0}$. Dash line: clean case; solid line: $l=a$ and $\xi=0.1$.

$(a / \xi)$ the prefactor is rather small. This weak suppression of the averaged susceptibilities can be related to the fact that in the long range case, different sectors of the contributing periodic orbits are highly correlated. As seen in Fig. 5(a), the quantum mechanical results (squares) for $\chi^{(t)}$ at $\xi / a=0.5$, which are closest to the long range case, exhibit already a very weak damping.

\section{B. Disorder effects at finite $\boldsymbol{H}$-field: From integrable to chaotic behavior}

In Fig. 6 we compare the ratio $\left(\chi^{(t)} / \chi^{0}\right)^{2}$ [obtained from calculating the integral in Eq. (5.1)] as a function of the dimensionless flux $\varphi=H a^{2} / \Phi_{0}$ for the clean case and for disorder characterized by $l=a$ and $\xi=0.1$. This figure shows that the damping due to disorder is maximal at zero field, but that already for $\varphi=5$ the disorder seems not to affect the magnetic response any further.

The origin of this behavior can be understood readily by observing that as soon as $\varphi$ is larger than one, the integral Eq. (5.1) is correctly approximated by a stationary phase approximation. ${ }^{5}$ The stationary point $x_{0}^{s}=a / 2$ corresponds to the two periodic orbits of the perturbed system, and only the trajectories such that

$$
\left(x_{0}-x_{0}^{s}\right)^{2} \varphi<1
$$

actually contribute to the integral. The magnetic field causes a de-phasing of the contributions of the various trajectories of the family, thus breaking the integrability of the system. This effect is responsible for the overall decrease of the typical susceptibility as the field increases. In this respect clean and disordered square billiards are not equivalent. In the disordered case, trajectories separated by a distance larger than $\xi$ are already not contributing in phase. Therefore the additional magnetic field affects the magnitude of the susceptibility much less. This remains true up to the point where the condition (5.11) implies $\left|x_{0}-x_{0}^{s}\right|<\xi$ in which case the disorder is not effective anymore, and the two curves coincide.

Therefore the behavior of the disorder damping we discussed in the previous subsection is characteristic for integrable geometries. For chaotic systems diagonal contributions (pair products of the same periodic orbit) are barely affected by disorder. This behavior is similar to that of integrable systems at finite field. When evaluating the contribution to the trace of the Green function in the neighborhood of a periodic orbit by stationary phase approximation, (as for the 
derivation of the Gutzwiller trace formula) only orbits extremely close to the periodic orbit under consideration actually contribute. Unless $\xi$ is exceedingly small, all these trajectories will see the same disorder potential.

As a final remark, note that non-diagonal contributions (pairs of different paths) are fully damped upon impurity average for chaotic as well as integrable systems, since the disorder potential along two different trajectories is usually not correlated [see also the discussion of the averaged Green function product after Eq. (3.17)]. Therefore non-diagonal contributions, which may be necessary to consider in the clean case, ${ }^{30}$ are exponentially suppressed in the presence of disorder. On the contrary, diagonal terms which contain orbit correlations on distances $\xi$, exhibit non-exponential behavior [Eq. (5.7)] as a function of the inverse MFP $1 / l$ for integrable geometries and are not affected (within our approximations) by disorder in the chaotic case.

\section{Relation to experiment and other theories}

Measurements of the orbital magnetism of small microstructures are still rare today. The only experiment on ensembles of ballistic billiards that we are aware of, was performed by Lévy et al. ${ }^{21}$ and investigated the magnetic susceptibility of an array of about $10^{5}$ ballistic square-like cavities. The size of the squares is on average $a=4.5 \mu \mathrm{m}$, with a large dispersion (estimated between $10 \%$ and 30\%) along the array. Each individual square is a mesoscopic ballistic system since the phase-coherence length is estimated to be $L_{\Phi}=15-40 \mu \mathrm{m}$ and the elastic mean-free-path $l=4.5-10 \mu \mathrm{m}$. The potential correlation length can be estimated ${ }^{24}$ to be of the order of $\xi / a \simeq 0.1$. Taking the most unfavourable case of $l \simeq a \simeq 4.5 \mu \mathrm{m}$ we obtain, with respect to the clean case, a disorder reduction for the averaged susceptibility of $\langle\bar{\chi}\rangle / \bar{\chi} \simeq 0.37$, showing that the features of the clean integrable systems (strong paramagnetic susceptibility at $H=0$ ) persist upon inclusion of disorder. Since $\bar{\chi} \simeq 100 \chi_{L},{ }^{5-7}$ our calculations for the paramagnetic response of the ballistic squares agree quantitatively with the experimental findings (given the experimental uncertainties).

Persistent currents in individual quasi-ballistic rings have recently been measured. ${ }^{31} \mathrm{~A}$ similar setup would be needed for measuring the magnetic response of singly connected geometries, where our typical susceptibility (5.1) should be measured for the integrable case. Since modern lithographic techniques allow one to design chaotic as well as integrable cavities ${ }^{2,3}$ and since we have demonstrated that disorder does not mask this difference, an order-of-magnitude effect is expected in the susceptibility according to the shape (chaotic vs. integrable) of the cavity.

In a related theoretical work Gefen et al. ${ }^{32}$ followed a complementary approach to ours and calculated the disorder-averaged susceptibility for an ensemble of ballistic squares based on long trajectories (strongly) affected by scattering from $\delta$-like impurities. They found that the average susceptibility does not depend on the elastic MFP. These results are not borne out by either our analytical or our semiclassical calculations at temperatures relevant for the experiment, where the exponential damping from Eq. (2.18) makes very long trajectories irrelevant.

\section{SUMMARY}

In this work we have studied the interplay between integrability and disorder in the ballistic regime. The integrable property of the confining potential of a microstructure implies a peculiar behavior of its thermodynamical response functions, like the magnetic susceptibility. The disorder effects provided by remote impurity scattering tend to weaken the importance of the boundary scattering (and therefore the relevance of the underlying classical mechanics). Using a semiclassical approach we quantify this damping and show it to be much weaker than previously estimated (power-law suppression instead of exponential damping for the typical and average susceptibility). The disorder damping is decisively affected by finite-size effects since it depends not only on bulk-like characteristics of the disorder (like the elastic mean-free-path), but also on the ratio between the size of the structure and the correlation length of the potential. 
TABLE I. Summary of the different average susceptibilities (at $H=0)$ considered in the short range $\left(\xi<\lambda_{F}<a\right)$, finite range $\left(\lambda_{F}<\xi<a\right)$ and long range $\left(\lambda_{F}<a<\xi\right)$ regimes. The fixed-size impurity averaged susceptibility $\langle\chi\rangle$ is given by the one-particle Green function, while the typical $\chi^{(t)}$ and average $\langle\bar{\chi}\rangle$ susceptibilities are given by two-particle Green functions and involve impurity and energy averages. The different average susceptibilities are normalized with respect to the corresponding clean counterparts. $L_{11}$ is the length of the shortest flux-enclosing periodic trajectories in the square. In the short range regime the damping is governed by the elastic mean-free-path $l_{\delta}$ given by the quantum mechanical expression (A4). The damping in the finite and long range regimes is governed by the elastic MFP $l$ [whose semiclassical expression is given in Eq. (3.14)], the correlation length $\xi$ of the impurity potential and the size $a$ of the structure. $I_{t}$ is the moment of inertia of the (11) trajectories [Eq. (3.16)]. The finite-range expressions for $\chi^{(t)}$ and $\langle\bar{\chi}\rangle$ showing a power-law damping hold in the deep ballistic limit $l<L_{11}$. The numerical factors are $c_{t}=(20 / 7) \sqrt{2 \pi}, c_{a}=2 \sqrt{2 \pi}, d_{1}=1 / 4 \sqrt{\pi}$, and $d_{2}=6.5 \cdot 10^{-5}$.

\begin{tabular}{llll}
\hline \hline & Short range & \multicolumn{1}{c}{ Finite range } & \multicolumn{1}{c}{ Long range } \\
\hline$\langle\chi\rangle / \chi_{c l}$ & $\exp \left(-L_{11} / 2 l_{\delta}\right)$ & $\exp \left(-L_{11} / 2 l\right)$ & $\exp \left\{-d_{1}\left(L^{2} / l \xi\right)\left[1-I_{t} /\left(2 \xi^{2}\right)\right]\right\}$ \\
$\left(\chi^{(t)} / \chi_{c l}^{(t)}\right)^{2}$ & $\exp \left(-L_{11} / l_{\delta}\right)$ & $c_{t}(\xi / a)\left(l / L_{11}\right)^{1 / 2}$ & $1-d_{2} a / l(a / \xi)^{9}$ \\
$\langle\bar{\chi}\rangle / \bar{\chi}$ & $\exp \left(-L_{11} / l_{\delta}\right)$ & $c_{a}(\xi / a)\left(l / L_{11}\right)^{1 / 2}$ & $1-d_{2} a / l(a / \xi)^{9}$ \\
\hline \hline
\end{tabular}

Our finding for the weak disorder damping is particularly important due to the large phase coherence effects found for clean integrable structures and to the fact that the difference in the magnetic response between integrable and chaotic geometries has not yet been experimentally demonstrated.

Our calculational tools have been semiclassical expansions, which naturally convey at the quantum level information about the underlying classical mechanics and its sensitivity with respect to disorder. For the weak disorder that we have considered in this work, the lowest order approximation consists of the perturbative modification of the classical actions by the impurity potential. Averages over impurity configurations following our semiclassical calculations, allow us to obtain various ensemble susceptibilities. Our analytical calculations have been checked against numerical quantum simulations performing exact diagonalizations of the corresponding Hamiltonian.

The need to consider different averages is inherent to ballistic nanostructures, which are sufficiently small to be non-self-averaging. These various types of impurity-averaged susceptibilities for integrable systems are summarized in Table I for the three regimes defined by the correlation length of the impurity potential. We have first studied the fixed-size averaged susceptibility, directly obtainable from the disorder average of one-particle Green functions. It corresponds to the case where different impurity configurations of a given sample with a fixed Fermi energy are considered. For the short range regime, where the disorder correlation length $\xi<\lambda_{F}$, we have an exponential suppression of the clean results governed by the short-range elastic mean-free-path $l_{\delta}$ and the length of the most relevant trajectories. This result also holds in the finite-range $\left(\lambda_{F}<\xi \ll a\right)$, but with an elastic mean-free-path that we have evaluated semi-classically. In the long-range regime $(\xi>a)$ the fixed-size averaged susceptibility depends exponentially on the product $(L / l) \cdot(L / \xi)$ (where $L$ denotes the typical orbit length) and a correction taking into account the geometry of the periodic trajectories.

For comparison with actual experiments we have to take into account that different impurity realizations are obtained together with a change in the Fermi energy and the size of the structures. We are then led to consider impurity and size averaged susceptibilities, which are expressed in terms of two-particle Green functions. The typical susceptibility is appropriate when considering the magnetic response of an individual sample which is thermally cycled in order to obtain different realizations of the potential. The average susceptibility is obtained from the measurement of an array of microscopically different samples. For the short-range case the only difference between one- and two-point Green function quantities is the factor $1 / 2$ of the exponential damping of the former. In the finite-range regime there appear important differences when considering two-point Green function quantities with respect to the one-particle case. Closed trajectories that 
remain closer than the correlation length of the potential result in a weak damping with a powerlaw dependence on $l / L$ and $\xi / a$. This is the experimentally relevant situation, and the use of standard parameters led us to conclude that disorder damping in currently realizable microstructures is sufficiently weak in order not to mask the large effects due to integrability. In the longrange case the damping due to disorder is extremely small.

We have further considered the interplay between disorder and magnetic field in integrable geometries. It is interesting to note that both have a similar effect since they produce de-phasing between nearby trajectories. Since the two sources of de-phasing do not superpose, we find that disorder is less effective at finite fields, and reciprocally, disordered samples are less sensitive to magnetic field.

In chaotic geometries periodic trajectories are usually isolated, resulting in smaller oscillations of the density of states and a much smaller magnetic response than integrable systems. Introduction of disorder in chaotic geometries is therefore less dramatic than in integrable systems, since it merely changes the action of the relevant periodic trajectories instead of producing de-phasing within a family. The transition from the ballistic regime (where classical trajectories are essentially unaffected by disorder) to the diffusive regime will be considered in a subsequent publication.

In this work we have started from a system that is physically realizable using modern technology and we have developed a theoretical model with some key ingredients involving integrability and disorder. These are deep theoretical issues that need to be complemented by the consideration of other effects, like interactions, in order to obtain a complete description of the thermodynamics of mesoscopic systems.

\section{ACKNOWLEDGMENTS}

We would like to thank Harold Baranger for numerous discussions and Paul Walker for a careful reading of the manuscript. K.R. and R.A.J. acknowledge support from the "Coopération CNRS/DFG', (EB/EUR-94/41) and the French-German program PROCOPE.

\section{APPENDIX: RELATION BETWEEN SEMICLASSICAL AND QUANTUM MECHANICAL RESULTS FOR BULK MEAN FREE PATHS}

It is instructive to compare the semiclassical results of Eqs. (3.12)-(3.14) for the ballistic regime with their counterparts obtained from quantum mechanical scattering theory.

In a perturbative diagrammatic approach (treating the related Dyson-equation for scattering within a self-consistent Born approximation) the damping of the disorder-averaged one-particle Green function in a random potential is of the same exponential form as in Eq. (3.11). ${ }^{9}$ This is usually obtained by replacing the imaginary part of the self-energy in the Green function after impurity average by the product of the density of states of the unperturbed system and $n: u^{2}$. The resulting quantum mechanical inverse elastic MFP $l_{\mathrm{qm}}$, which appears in Eq. (3.11), is related to the total cross section $\sigma$ by means of

$$
\frac{1}{l_{\mathrm{qm}}}=n_{i} \sigma,
$$

where $n_{i}$ is the impurity density and

$$
\sigma=\int \mathrm{d} \Theta \sigma(\Theta)
$$

with $\sigma(\Theta)$ being the partial cross section for scattering with an angle $\Theta$.

For a Gaussian disorder potential of the form of Eq. (3.3) a calculation of the cross section can be performed analytically and the corresponding inverse MFP gives 


$$
\frac{1}{l_{\mathrm{qm}}}=\frac{1}{l_{\delta}} I_{0}\left[2(k \xi)^{2}\right] e^{-2(k \xi)^{2}}
$$

Here, $I_{0}$ is a modified Bessel function and

$$
\frac{1}{l_{\delta}}=\frac{2 \pi}{\hbar} \frac{n_{i} u^{2}}{v_{\mathrm{F}}} d(\mu)=\frac{n_{i} u^{2}}{v_{\mathrm{F}}} \frac{m}{\hbar^{3}}
$$

is the inverse MFP for the white noise case of $\delta$-like scatterers of mean strength $u$. The $v_{\mathrm{F}}$ is the Fermi velocity and $d(\mu)=m /\left(2 \pi \hbar^{2}\right)$ the density of states at the Fermi energy of a 2DEG. ${ }^{9}$

In order to compare $l_{\mathrm{qm}}$ with our semiclassical result we expand $l_{\mathrm{qm}}(k \xi)$ for large $k \xi$ which gives

$$
l_{\mathrm{qm}}(k \xi) \simeq \sqrt{4 \pi}(k \xi) l_{\delta}\left[1-\frac{1}{16(k \xi)^{2}}\right] \text { for } k \xi \rightarrow \infty .
$$

The leading order term is exactly the semiclassical MFP Eq. (3.14) for the Gaussian disorder model (3.3). The agreement between the semiclassical and diagrammatic approaches for the bulk can be related to the fact that our semiclassical treatment of disorder corresponds to the use of the Eikonal approximation (for each single scattering event) which is known to give the same results as Born approximation for large $k \xi$.

In the limit of $\xi<\lambda_{F}$ where our semiclassical description is no longer applicable, the mean free path $l_{\mathrm{qm}}$ approaches $l_{\delta}$, which means that Eq. (3.11) can further be used, but with the semiclassical $l$ replaced by $l_{\delta}$.

The quantum mechanical transport mean free path $l_{\mathrm{T}}$ is calculated by including a factor $(1-\cos \Theta)$ in the integral (A2) for the scattering amplitude. It reads for Gaussian disorder

$$
\begin{aligned}
\frac{1}{l_{\mathrm{T}}} & =\frac{1}{l_{\delta}}\left(I_{0}\left[2(k \xi)^{2}\right]-I_{1}\left[2(k \xi)^{2}\right]\right) e^{-2(k \xi)^{2}} \\
& \simeq \frac{1}{l_{\mathrm{qm}}} \frac{1}{4(k \xi)^{2}} \quad \text { for } k \xi \rightarrow \infty .
\end{aligned}
$$

This relation shows that $l_{\mathrm{T}}$ can be considerably larger than $l_{\mathrm{qm}}$ for $\lambda_{F}<\xi$. This shows that in the case of a confined system and smooth disorder, the system may behave ballistically although the elastic MFP $l$ state might be considerably smaller than the system size.

\footnotetext{
${ }^{1}$ H.U. Baranger, R.A. Jalabert, and A.D. Stone, Chaos 3, 665 (1993).

${ }^{2}$ C.M. Marcus, R.M Westervelt, P.F. Hopkings, and A.C. Gossard, Chaos, 3, 643 (1993).

${ }^{3}$ A. M. Chang, H.U. Baranger, L. N. Pfeiffer, and K. W. West, Phys. Rev. Lett. 73, 2111 (1994).

${ }^{4}$ M.C. Gutzwiller, Chaos in Classical and Quantum Mechanics (Springer, Berlin, 1990).

${ }^{5}$ D. Ullmo, K. Richter, and R.A. Jalabert, Phys. Rev. Lett. 74, 383 (1995).

${ }^{6}$ K. Richter, D. Ullmo, and R.A. Jalabert, Phys. Rep. (in print, 1996).

${ }^{7}$ F. von Oppen, Phys. Rev. B 50, 17151 (1994).

${ }^{8}$ S. Das Sarma and F. Stern, Phys. Rev. B 32, 8442 (1988).

${ }^{9}$ A.A. Abrikosov, L.P. Gorkov, and I.E. Dzyaloshinski, Methods of Quantum Field Theory in Statistical Physics (PrenticeHall, Englewood Cliffs, 1963)

${ }^{10}$ K. Efetov, Adv. Phys. 32, 53 (1983).

${ }^{11}$ H.U. Baranger and P. Mello, Phys. Rev. Lett. 73, 142 (1994)

${ }^{12}$ R.A. Jalabert, J.-L. Pichard, and C. W. J. Beenakker, Europhys. Lett. 27, 255 (1994).

${ }^{13}$ Z. Pluhar, H. A. Weidenmüller, J. A. Zuk, and C. H. Lewenkopf, Phys. Rev. Lett. 73, 2115 (1994).

${ }^{14}$ K. Efetov Phys. Rev. Lett. 74, 2299 (1995).

${ }^{15}$ B. L. Altshuler and B. I. Shklovskii, Zh. Éksp. Teor. Fiz. 91, 220 (1986) [Sov. Phys. JETP 64, 127 (1986)].

${ }^{16}$ K. Richter, D. Ullmo, and R.A. Jalabert, Phys. Rev. B 56, R 5219 (1996).
} 
${ }^{17}$ A. Schmid, Phys. Rev. Lett. 66, 80 (1991); F. von Oppen and E.K. Riedel, ibid. 66, 84 (1991); B.L. Altshuler, Y. Gefen, and Y. Imry, ibid. 66, 88 (1991).

${ }^{18}$ Y. Imry, in Coherence Effects in Condensed Matter Systems, edited by B. Kramer (Plenum, New York, 1991).

${ }^{19}$ M.V. Berry, and M. Tabor, Proc. R. Soc. London, Ser. A 349, 101 (1976).

${ }^{20}$ A.M. Ozorio de Almeida, in Quantum Chaos and Statistical Nuclear Physics, edited by T.H. Seligman and H. Nishioka, Lecture Notes in Physics 263 (Springer, Berlin 1986).

${ }^{21}$ L.P. Lévy, D.H. Reich, L. Pfeiffer, and K. West, Physica B 189, 204 (1993).

${ }^{22}$ A. Altland and Y. Gefen, Phys. Rev. B 51, 10671 (1995).

${ }^{23}$ We do not aim to describe disorder within a microscopic model as for example that by Nixon and Davies (J.A. Nixon and J.H. Davies, Phys. Rev. B 41, 7929 (1990)), where calculations for realistic distributions of residual impurities in a semiconductor heterostructure are performed.

${ }^{24}$ Self-consistent calculations indicate that the characteristic scale $\xi$ can be in the order of 100-200 nm, A.M. Zagoskin et al. (preprint, Cond-Mat/9404077).

${ }^{25}$ A. D. Mirlin, E. Altshuler, and P. Wölfle (preprint, Cond-Mat/9507081).

${ }^{26} \mathrm{~A}$ mixed approach for the diffusive regime where the individual scattering is treated quantum mechanically and the propagation between collisions (with $\delta$-like scatterers) in terms of semiclassical paths can be found in S. Chakravarty and A. Schmid, Phys. Rep. 140, 193 (1986).

${ }^{27}$ For diagrammatic approaches to disorder effects on the susceptibility of small magnetic particles see S. Oh, A. Yu. Zyuzin, and A. Serota, Phys. Rev. B 44, 8858 (1991); A. Raveh and B. Shapiro, Europhys. Lett. 19, 109 (1992); B.L. Altshuler, Y. Gefen, Y. Imry, and G. Montambaux, Phys. Rev. B 47, 10340 (1993).

${ }^{28}$ S. Doniach and E. H. Sondheimer, Green's Functions for Solid State Physicists (Addison-Wesley, Reading, 1978).

${ }^{29}$ That is, we implicitly assume that spatial fluctuations in the impurity potentials $V_{\alpha}(\mathbf{r})$ are independent of $V$. This may lead to an overestimation of impurity effects for configurations with extreme values of $\bar{V}$ which are however of exponentially small number.

${ }^{30} \mathrm{~A}$ proper treatment of these non-diagonal terms in the clean limit is an outstanding problem in the context of semiclassical quantum transport.

${ }^{31}$ D. Mailly, C. Chapelier, and A. Benoit, Phys. Rev. Lett. 70, 2020 (1993).

${ }^{32}$ Y. Gefen, D. Braun, and G. Montambaux, Phys. Rev. Lett. 73, 154 (1994). 